\title{
Wavelet Robust Filtering of Out-trajectory Data
}

\author{
Lingjing Meng \\ Section 94, Unit 91550 \\ Dalian, China \\ menglingj@yahoo.com.cn
}

\author{
Haibo Liu \\ Section 94, Unit 91550 \\ Dalian, China \\ hunter24099@163.com
}

\begin{abstract}
Wavelet-based robust filtering of process data is proposed in order to reduce the influence of the outliers and noise in Out-trajectory data. We utilize the moving median filtering method to reject outliers in the original data and then combine wavelet de-noising method with empirical Wiener threshold to suppress noise. Simulation calculation and real engineering application has shown that the novel algorithm reliably preserves the information encapsulated in a process signal corrupted with noise and outliers. The methodology has been proved to be reliable and robust.
\end{abstract}

Keywords-wavelet transform; empirical wiener Filtering; moving median

\section{INTRODUCTION}

The aircraft Out-trajectory measurement system with a large deviation of outliers often produce outliers for many reasons, equipment error or data record and interpretation process [1], the sudden change and interference of the surrounding environment, as well as the fault of the operator. Therefore, it is very difficult to find a perfect discrimination and processing method to correct the outliers. The observation data which often comprises outliers and noise signal leads to serious distortion, reduce the value of the degree of confidence of the observation data, Therefore, you must first reject outliers in observational data, find reasonable, credible data to replace it, and then try to suppress noise, to ensure the accuracy of the outside measurement processing results. Therefore, excluding outliers and noise suppression has been an important research area.

Classic filter de-noising algorithm processing, such as polynomial filtering, smoothing and differential method, as well as the observation model-based methods such as Kalman filtering outliers are very sensitive[1]. Theoretical analysis and actual validation results confirm that, when the measurement data contain a small amount of outliers will lead to a serious distortion or even collapse [2].

Donoho and Johnstone proposed a method for reconstructing unknown process data where they employed thresholding in the wavelet domain and showed this to be asymptotically near optimal for a wide class of process data corrupted by additive white Gaussian noise (AWGN)[3, 4]. Since wavelet techniques offer a different view of data than those presented by traditional techniques, wavelet analysis can often compress or de-noise process data without significant degradation, even when one wants to preserve both high and low frequency components. 1999 Bakhtazad
[5] proposed wavelet transform coefficient de-noising (coefficient de-noising) method by processing a series of test signal, it is proved that this method is superior to the traditional wavelet de-noising algorithm, but this method is not robust, when the signal contains outliers, the filtering effect will seriously deteriorate. In recent years, Chinese scholars have similar research [6-9], but less concentrate in complex signals containing both outliers and noise, and the robustness of the algorithm are not deep enough causing some engineering difficult in practical application.

This article will move in the value of the (moving median, hereinafter referred to as MM) filtering algorithm combined filtering algorithm based on wavelet transform experience Wiener threshold (empirical Wiener thresholding), first moving median filtering algorithm, excluding the original data outliers Then use the wavelet coefficient de-noising algorithm combines the experience Wiener threshold filtering algorithm proposed in this paper, to suppress the noise in the data. The simulation analysis and practical test show that this method has good robustness can effectively eliminate anomalies in the data, the noise immunity application conditions and wide adaptability.

\section{WAVELET DE-NOISING METHODS}

\section{A. Conventional wavelet domain de-noising}

Conventional wavelet domain de-noising methods are based on taking the discrete wavelet transform of a signal, passing the corresponding coefficients through a threshold step which removes wavelet coefficients below a certain value. In Fig. 2, the process signal is contaminated with noise $x[i]=s[i]+n[i]$, where $x[i]$ is the signal of interest and $n[i]$ is noise. Through wavelet transform coefficients model is $y[i]=u[i]+z[i], y=W x, u=W s, z=W n$.

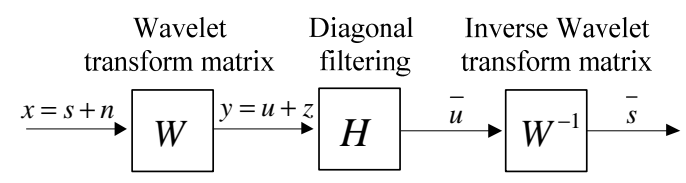

Figure 1. Wavelet-based filtering

Donoho(1993) suggested the following non-linear threshold methods:

1. Hard threshold filter $H_{h}=\operatorname{diag}\left[h_{h}(1), h_{h}(2), \ldots, h_{h}(N)\right]$ discards coefficients below a threshold $\tau$ 


$$
h_{h}(i)= \begin{cases}1, & |y(i)|>\tau \\ 0, & |y(i)| \leq \tau\end{cases}
$$

2. Soft threshold filter $H_{s}=\operatorname{diag}\left[h_{s}(1), h_{s}(2), \ldots, h_{s}(N)\right]$ is similar, shrinks the wavelet coefficients above the threshold

$$
h_{s}(i)= \begin{cases}\frac{\operatorname{sgn}[y(i)] \cdot[|y(i)|-\tau]}{y(i)}, & |y(i)|>\tau \\ 0, & |y(i)| \leq \tau\end{cases}
$$

\section{B. Wavelet domain Wiener de-noising}

The optimal Wiener filter is an operator on the noisy input data that can extract the desired signal from the input. The Wiener filter can be optimal by assuming that the input and output sequences of the data are mutually wide sense stationary from a statistical viewpoint (Papoulis, 1977). Furthermore, the Wiener filter requires the primary knowledge of statistics of the desired signal as well as the noise.

Optimum Wiener filters approximation can be carried out by the wavelet transform. We can consider this approximation of the optimum Wiener filter as a nonlinear shrinkage of wavelet coefficients. Therefore, Wiener Shrink refers to minimizing the mean square error estimation of the desired signal coefficients among the contaminated ones by an approximation of the Wiener filter as a diagonal filter.

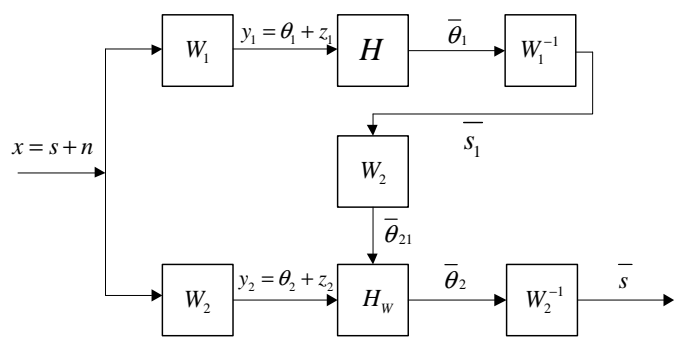

Figure 2. Wiener filtering

\section{ROBUST FILTERING}

A robust filtering method is proposed in this paper which can reject outliers in the original data and then combine wavelet de-noising method with empirical Wiener threshold to suppress noise.

For de-noising, there are efficient methods that can accommodate noise with various distributions as well as different signal characteristics. Bakhtazad, Palazoglu, and Romagnoli (1999) proposed a coefficient de-noising approach and proved that it outperforms many filtering techniques on benchmark process signals. Nevertheless, this approach is not robust, hence, when applied to a signal contaminated with outliers, its performance substantially deteriorates. Therefore, we propose a strategy that uses the MM filter in tandem with the coefficient de-noising approach to address this problem in an effective manner. The coefficient de-noising uses Wiener thresholding due to its optimality.
MM filter was developed by Tukey (1970) and has been successfully applied in many areas of signal estimation. The MM filter is used only to treat the outliers so that the coefficient de-noising technique can deal with the noise artifacts effectively [10]. It should be noted also that the MM not only treats the outliers present in the signal but also rejects the noise to some extent. Hence, his feature of the MM filter complements the coefficient de-noising technique by improving its performance considerably even though the signal may be contaminated with noise only.

\section{A. Algorithm}

- In the actual data processing, in order to remove the outliers on the subsequent de-noising algorithm, firstly using $\mathrm{MM}$ filter proposed outliers in the original data, MM filter is defined as:

$$
f_{m}[n]=\operatorname{med}(f[n-(w-1) / 2], \ldots, f[n+(w-1) / 2])
$$

- Most of the more stable signal energy is concentrated on the details of the signal, bigger threshold will lose a lot of energy, but also some useful information is filtered out, the threshold value is too small and would not achieve the effect of removing noise, in order to solve this contradiction, using wavelet transform twice $W^{2}$, reducing energy distribution on the detail coefficients.

- The experience Wiener filter algorithm $H_{w}=\operatorname{diag}\left[h_{w}(1), h_{w}(2), \ldots, h_{w}(N)\right], h_{w}(i)=\bar{\theta}_{21}^{2}(i) /\left[\bar{\theta}_{21}^{2}(i)+\bar{\tau}^{2}\right]$ Details are shown in Fig.2

- Right after the inverse wavelet transform of the filtered data twice reconstructed signal can be obtained by the removal of outliers and noise signal.

The overall process flow is shown in Figure 3:

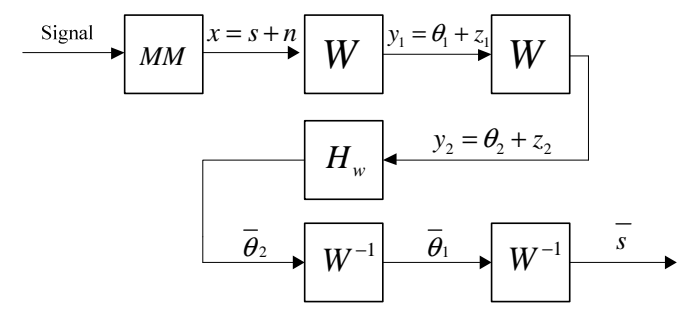

Figure 3. Overall process flow is shown

\section{B. Simulation Analysis}

To investigate the behavior of our proposed strategy on noise and/or outlier rejection, four benchmark signals have been tested. These standard signals are Doppler, Blocks, Bumps and HeaviSine and taken from wavelet toolbox.

We added noise and outliers to these signals and applied the robust de-noising strategy (moving median filter plus coefficient de-noising). The additive Gaussian white noise statistics are given by $\mathrm{N}(0,1)$. The outliers were generated from Poisson distribution and distributed along the signal randomly. For thresholding, WienerShrink was used as a nonlinear shrinkage of the wavelet coefficients. 
The proposed algorithm filter can be seen from Fig 4-7, the output of the signal with the original signal as well, the algorithm effectively removed outliers and noises for four types of signals benchmark. showed good adaptability (see Table 2), with the outliers from 30 to 100, the paper proposes $\mathrm{MM}+$ coefficient filtering algorithm variance does not significantly increase, and the results are better than the table listed the other algorithms, indicating that the algorithm is robust.

Residuals results are often larger within the intense extreme fluctuations area (such as the first half of Doppler). In this area outliers is very similar with the real signal resulting in MM method output outliers; but within the relatively flat part, outliers and de-noising suppression effect significantly improved The result has shown that there is still some room for algorithm of this article to improve in extreme fluctuations area.
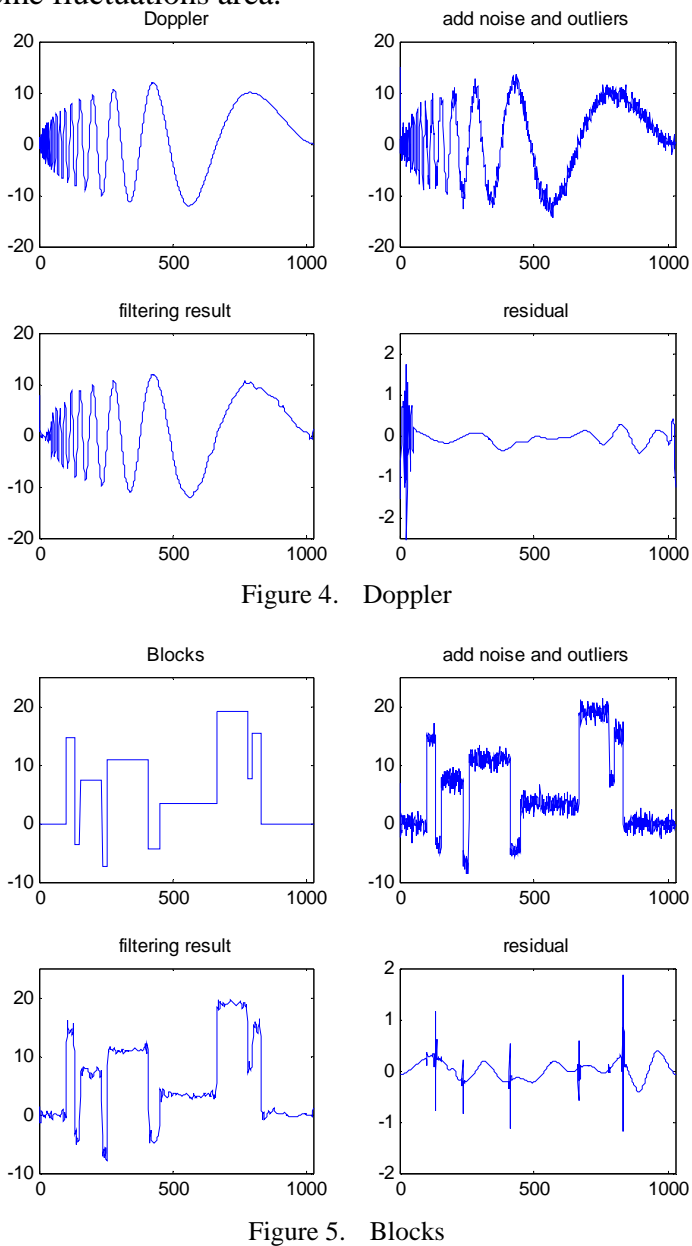
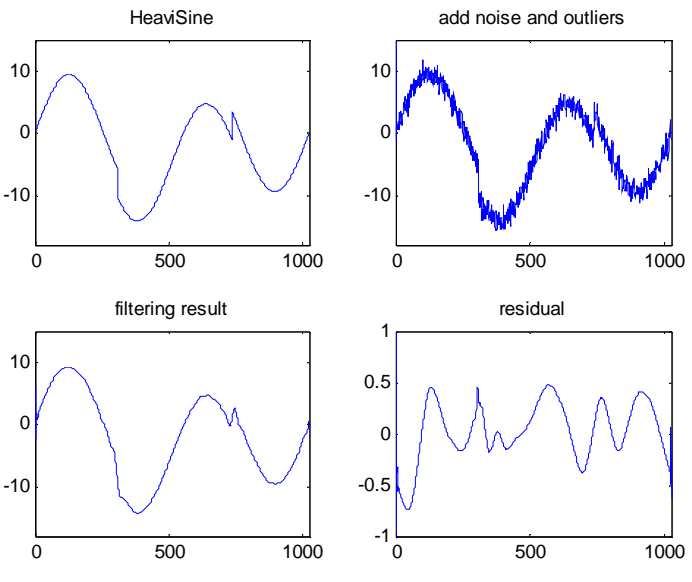

Figure 6. HeaviSine
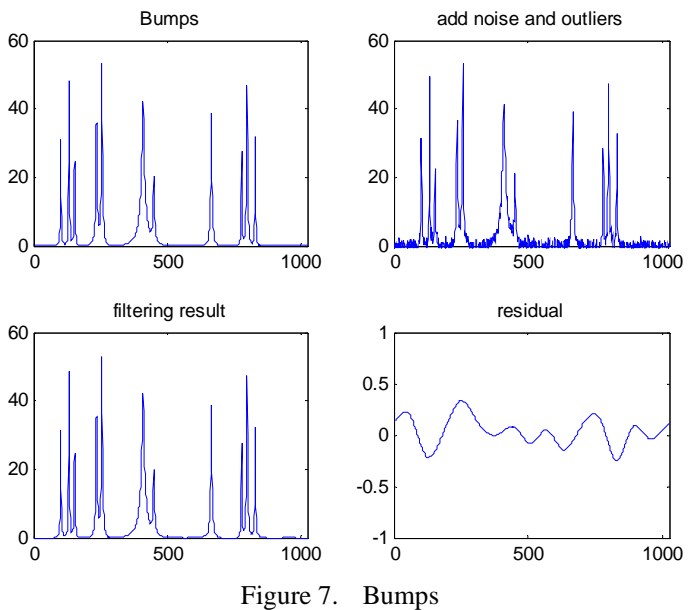

Filter parameters window size of the MM filter, and decomposition level of the wavelet-based filters are given in Table I. The filter uses 'daubechies' basis function with eight vanishing moments. We selected the window size such that the sole purpose of the MM filter is to reject outliers and not to suppress the noise. The decomposition level of the wavelet filters, on the other hand, is kept at the smallest magnitude level.

TABLE I. FILTER PARAMETERS USED IN MM AND WAVELET-BASED FILTERS

\begin{tabular}{|c|c|c|}
\hline Signal & Window size & Decomposition level \\
\hline Doppler & 5 & 4 \\
\hline Blocks & 5 & 2 \\
\hline HeaviSine & 5 & 5 \\
\hline Bumps & 3 & 3 \\
\hline
\end{tabular}

The results shown in Table II were obtained by averaging the MSE of 2000 trials. As expecting, the performance of the wavelet filters deteriorated significantly due to the outliers. The poor performance of the wavelet filters was not unexpected because the outliers fall into the low frequency range of the original signal, hence their effect appears in the approximation part of the signal decomposition. However, for the combined strategy of the MM filter and wavelet filters, 
the performance has notably improved in the MSE of estimation.

\section{Engineering Application}

The results shown in Fig 8: top left is the original signal; upper right lower left for the removal of the signal after the noise; through MM filtering to remove the result of the outliers; lower right for the residual. Processing results show that the filtering method proposed in this paper successfully removed the outliers in the raw data, and to a considerable extent on the suppression of noise more clearly reveal the characteristics of the movement of the aircraft in different time periods, laid good foundation for subsequent analysis.
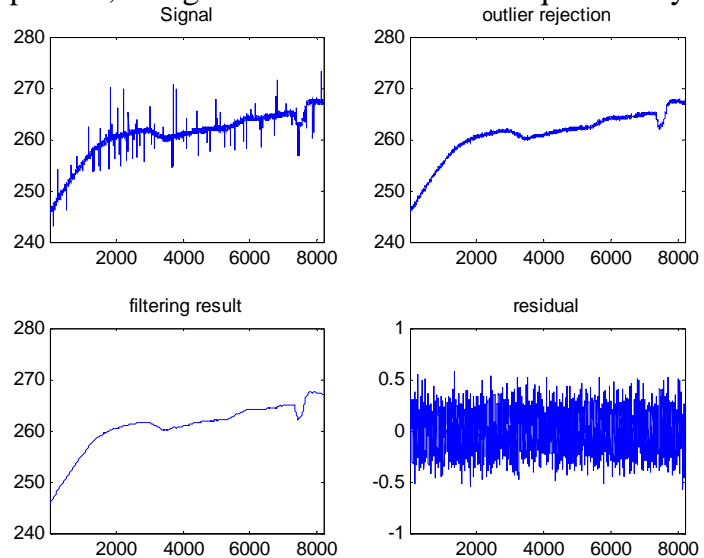

Figure 8. Real task filtering result

\section{CONCLUSIONS}

The proposed robust filtering method (moving median plus coefficient de-noising) is shown to have great effect on noise and outlier suppression. Four typical benchmark signal and the actual task of measurement data is proceed and analyzed, the results has shown that the filtering method proposed in this paper can not only effectively eliminate outliers in the raw data but also suppress the noise, Good results are obtained in the simulation and practical engineering applications, so there are potential prospects for the application in measurement data processing.

\section{REFERENCES}

[1] L. Li-sheng, Out-trajectory measurement data processing, Beijing: Industrial national defense press, 2002.

[2] Z. Lian-feng, Y. Xiao-feng and M. Zhi-hua, "An Integral and Recursive Filtering Method for Truncation Error Minimization and Outlier Suppression", Journal of Spacecraft TT\&C Technology, vol. 25, 2006, pp. 85-90.

[3] D.L. Donoho, De-noising by soft thresholding, "Ieee $\mathrm{T}$ Inform Theory", vol. 41, 1995, pp. 613-627.

[4] D.L. Donoho and I.M. Johostone, Ideal spatial adaptation via wavelet shrinkage, "Biometrica", vol. 81, 1994, pp. 425-455.

[5] A. Bakhtazad, A. Palazoglu and J. Romagnoli, Process data denoising using wavelet transform, "Intelligent Data Analysis", vol. 4, 1999, pp. 267-285.

[6] W. Kui-nai, Z. Zhu-rong and L. Xue-feng, "Study on Application of Wavelet Filters in Out-trajectory Data Processing", Journal of Spacecraft TT\&C Technology, vol. 25, 2006, pp. 72-76.

[7] G. Rao, M. Qing-Ci and A. Yu-hua, "A Method of Removing the Outliers in Data Processing of Miss-distance Based on Wavelet Transform", Journal of Spacecraft TT\&C Technology, vol. 25, 2006, pp. 64-67.

[8] L. Heng-bing, H. Shi-heng and L. Bing, "Wavelet de-noising based on novel thresholding function and best decomposition scale", Computer Engineering and Applications, vol. 43, 2007, pp. 73-74.

[9] D. Hao-pan and C. Shuang, "The Study on Wavelet De-noising under the Environment of MATLAB", Computer Simulation, vol. 20, 2003, pp. 119-122.

[10] J.W. Tukey, Exploratory data analysis. Reading ,MA: AddisonWesley, 1970.

TABLE II. MSE OF ESTIMATION FOR BENCHMARK SIGNALS ORRUPTED WITH N(0,1) AGWN AND 20 \& 100 OUTLIERS

\begin{tabular}{|c|c|c|c|c|c|c|c|c|}
\hline \multirow[b]{2}{*}{ Outliers number } & \multicolumn{2}{|c|}{ Doppler } & \multicolumn{2}{|c|}{ Blocks } & \multicolumn{2}{|c|}{ HeaviSine } & \multicolumn{2}{|c|}{ Bumps } \\
\hline & 30 & 100 & 30 & 100 & 30 & 100 & 30 & 100 \\
\hline MM filtering & 0.3375 & 0.4252 & 0.3489 & 0.4809 & 0.2984 & 0.3659 & 0.8755 & 1.3789 \\
\hline Wiener thresholding & 5.5391 & 20.2354 & 5.6598 & 19.6743 & 5.3356 & 20.4586 & 6.5663 & 19.7874 \\
\hline Coefficient de-noising & 4.6086 & 15.8771 & 5.0745 & 17.5446 & 6.0981 & 21.3445 & 6.0988 & 14.5561 \\
\hline MM filtering + Wiener thresholding & 0.2347 & 0.2743 & 0.3245 & 0.4103 & 0.2734 & 0.3134 & 0.7891 & 1.2343 \\
\hline MM filtering + Coefficient de-noising & 0.1346 & 0.1561 & 0.2681 & 0.3512 & 0.1098 & 0.1167 & 0.5981 & 0.9943 \\
\hline
\end{tabular}

